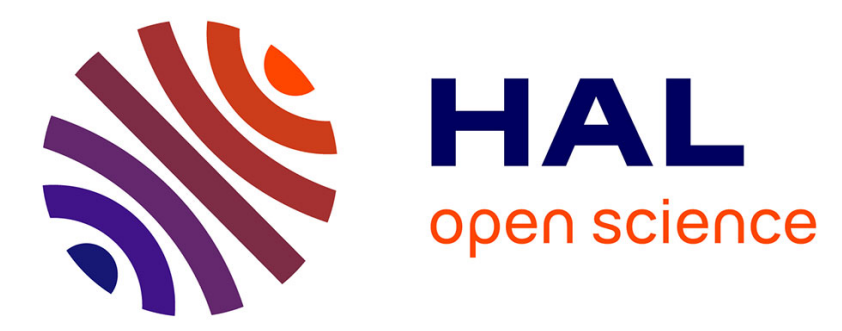

\title{
Comparer a posteriori. Retour sur une expérience collective de recherche
}

Marie-Laure Geoffray, Amélie Le Renard, Soline Laplanche-Servigne

\section{To cite this version:}

Marie-Laure Geoffray, Amélie Le Renard, Soline Laplanche-Servigne. Comparer a posteriori. Retour sur une expérience collective de recherche . Terrains et Travaux: Revue de Sciences Sociales, 2012. halshs-01726642

\section{HAL Id: halshs-01726642 \\ https://shs.hal.science/halshs-01726642}

Submitted on 8 Mar 2018

HAL is a multi-disciplinary open access archive for the deposit and dissemination of scientific research documents, whether they are published or not. The documents may come from teaching and research institutions in France or abroad, or from public or private research centers.
L'archive ouverte pluridisciplinaire HAL, est destinée au dépôt et à la diffusion de documents scientifiques de niveau recherche, publiés ou non, émanant des établissements d'enseignement et de recherche français ou étrangers, des laboratoires publics ou privés. 


\section{Comparer a posteriori: retour sur une expérience collective de recherche}

\section{Introduction}

Lorsqu'on ne peut pas mener une étude comparative seul(e), peut-on néanmoins produire des résultats en comparant en équipe et a posteriori ? C'est le pari que nous avons fait au cours d'une expérience collective de recherche menée dans le cadre de doctorats en sociologie politique. Nous travaillions alors sur des contextes très différents, mais avions fait le choix de méthodes qualitatives, pour plusieurs d'entre nous d'inspiration ethnographique, avec une immersion longue dans les contextes étudiés et une perspective compréhensive qui accorde une place importante à la singularité des situations. Ce choix rendait nos approches relativement suspectes d'être inaptes à toute comparaison.

C'est notamment pour battre en brèche ce préjugé, mais aussi pour travailler selon un nouvel angle d'approche nos questions de recherche respectives que nous avons organisé une journée d'étude qui consistait à discuter des papiers comparatifs écrits à quatre mains. L'initiative s'inscrivait dans la continuité de dialogues entretenus depuis trois ans, au sein d'un groupe de travail, sur différentes thématiques qui nous rassemblaient et sur les points de convergence entre nos terrains d'enquête. Elle visait à pousser plus loin ces dialogues, à les systématiser, à en récolter les fruits et à en percevoir les limites. Concrètement, deux chercheurs ou chercheuses travaillant sur des objets proches dans des contextes différents devaient présenter en binôme le produit de leurs discussions comparatives concer- 
nant la construction de leur questionnement de recherche, la production du matériau de l'enquête et leurs interprétations et analyses. Cette démarche, que nous appelons comparatisme a posteriori, va bien au-delà des dialogues engagés au cours de colloques ou séminaires avec des collègues travaillant sur des objets proches. Le fait de devoir écrire une communication en binôme et de s'obliger à confronter de manière systématique deux enquêtes constitue une démarche spécifique.

Parmi les travaux consacrés aux approches comparatives en sciences sociales, le type de démarche que nous avons initiée n'a pas à notre connaissance fait l'objet de réflexions méthodologiques. D'une manière générale, la comparaison notamment en sociologie et en science politique est envisagée comme " une stratégie d'enquête et de recherche qui imprègne l'ensemble de la démarche du chercheur, de la définition de la problématique au choix du terrain, en passant par la construction des données, leur analyse et leur explication » (Vigour, $2005: 17$ ), que cette comparaison soit effectuée de manière individuelle ou collective. Or comparer a posteriori et en binôme inverse l'ordre des étapes de la démarche comparative tel qu'il est le plus souvent décrit. En effet, à l'exception de deux participantes dont l'expérience sera détaillée plus loin, nos objets et nos enquêtes ont été construits dans des perspectives principalement monographiques - même si toute démarche en sciences sociales est forcément comparative au sens où elle s'inscrit dans un champ de questionnements forgés à la lecture de travaux portant sur d'autres contextes.

Ce choix monographique nétait pas seulement lié à des contraintes matérielles (temps, connaissance de la langue). Nous ne nous reconnaissions pas totalement dans le comparatisme qualifié par Kocka (2003) d' " analytique » qui vise à contrôler et expliquer, à rechercher des causes, à trouver des " variables explicatives » pertinentes, à classer, ordonner et généraliser (Giraud, 2003 ; Vigour, 2005). Il n'est pas sûr que de tels objectifs puissent être atteints par le comparatisme a posteriori tel que nous l'avons envisagé, mais cela nétait pas notre but premier. Dans le cadre de nos recherches monographiques respectives, nous visions davantage à la mise en lumière de processus et/ou de trajectoires que de rapports de causalité. Nous étions notamment inspirées par les analyses localisées du politique (Briquet et Sawicki, 1989) et par des approches « par le bas » (Bayart, 1992 ; Revel, 1996). Dès lors, confronter nos recherches visait à partager des questionnements et à réfléchir aux concepts plus qu'à généraliser l'explication de tel phénomène par telle variable.

1. Lauteur identifie trois autres types de comparatisme : descriptif, heuristique, paradigmatique. 
Dans le présent article, nous revenons sur les étapes de ce travail collectif de comparaison a posteriori, de la constitution des binômes à l'émergence de questionnements communs, voire, pour certains binômes, d'hypothèses partagées. Puis nous montrons que même lorsque la démarche comparatiste n'a pas mené aux résultats attendus en termes de production commune, elle a considérablement enrichi les approches monographiques par la confrontation à des enquêtes, des regards et des interprétations différentes.

\section{Les binômes et leurs objets par ordre d'apparition dans le texte}

S'il est impossible d'entrer ici dans le détail de chaque expérience comparative en binôme, nous en mentionnerons certains aspects au fil de l'article afin d'entrer dans la " cuisine » de ce travail collectif ${ }^{1}$. Nous évoquerons seulement trois des sept binômes pour faciliter la lecture.

Binôme 1 : Les usages du shopping mall (centre commercial) dans le contexte de sociétés ségréguées (Port-Louis, Ile Maurice / Riyad, Arabie Saoudite)

Binôme 2 : Mobilisations de minorisés autour de l'enjeu de la mémoire de l'esclavage (Paris / Paris)

Binôme 3 : Espaces d'expression en milieu contraint (La Havane / Pékin)

1. Pour faciliter la lecture, les binômes seront évoqués à la troisième personne, y compris ceux auxquelles les auteures du texte appartenaient.

\section{La constitution des binômes : construire la comparabilité}

Dans le cas d'une comparaison décidée en amont de l'enquête, la détermination des objectifs et hypothèses participe au choix des terrains et des critères de comparabilité. Dans le cas du comparatisme a posteriori, cette étape est remplacée par celle du choix d'un partenaire dont l'enquête, déjà achevée, semblera sous un certain angle comparable à la sienne.

Le premier apport de la démarche découlait du fait qu'aucune comparaison nétait évidente : autrement dit, on ne pouvait pas tomber dans le piège de la « comparabilité spontanée " (Hassenteufel, 2000 : 109). Il s'agissait bien de construire des objets comparables en confrontant des matériaux d'enquête recueillis de manière autonome et dans des buts différents. Cela a été renforcé par le fait que les binômes, constitués pour la majorité d'entre eux sur la base d'une interconnaissance préalable, étaient relativement hétérogènes en termes de contextes, ce que nous envisagions da- 
vantage comme un défi que comme un obstacle. Au moins pour certaines d'entre nous travaillant sur des contextes réputés « exotiques » et " exceptionnels »- de manière flagrante pour les cas de Cuba, de la Chine et de l'Arabie Saoudite - notre envie de comparer avait été alimentée par une insatisfaction concernant le clivage entre une sociologie politique "générale », focalisée sur la France et l'Europe, et l'étude des " aires culturelles », souvent perçues comme plus « exotiques » et donc, implicitement, à portée généralisante plus réduite (Picard, 2006 : 5-11; Thiriot et al 2004). Rester confinées à une " aire culturelle » ne nous semblait pas heuristique. Il nous paraissait plus intéressant de confronter nos objets, nos enquêtes et nos résultats en ayant en tête l'audace comparative revendiquée par certains auteurs (Detienne, 2000 ; Veyne, 1976), consistant notamment à confronter des contextes a priori incomparables, tout en restant lucides sur le caractère expérimental de la démarche. Nous pensions que l'expérience serait enrichissante en soi sans forcément produire des résultats directement publiables. C'est d'ailleurs le fait de comparer des enquêtes déjà menées qui a concrètement rendu possible cette audace comparative : il est peu vraisemblable qu'un tel projet impliquant la comparaison de contextes habituellement pensés comme incomparables aurait été retenu par une agence de financement de la recherche.

Pour certains binômes, les sites où les enquêtes avaient été réalisées présentaient des similarités dans des contextes socio-politiques de prime abord très différents, comme dans le cas des shopping malls à Riyad et à PortLouis. C'est cette similarité de départ qui a poussé les deux chercheuses du binôme 1 à prendre l'initiative de confronter leurs matériaux, même si, dans leurs recherches respectives, elles avaient des méthodologies d'enquête et des questions de recherche (centrées sur le genre/le multiculturalisme) différentes (voir Le Renard, 2011 ; Peghini, 2009). Leurs matériaux semblaient compatibles parce qu'elles avaient recueilli des éléments à la fois sur les entrepreneurs et managers de ces entreprises, par entretiens, et sur les clients/usagers des shopping malls. La première avait étudié les usages de ces espaces en les fréquentant avec de jeunes Saoudiennes souvent rencontrées ailleurs (campus universitaire, lieux de travail) dans le cadre d'une enquête plus large d'inspiration ethnographique, tandis que la seconde avait procédé par questionnaires et observation. Leurs approches s'accordaient dans la mesure où elles étudiaient le mall dans son contexte social urbain - elles y avaient mené des enquêtes sur d'autres objets. Pour circonscrire la comparaison, elles se sont limitées aux deux shopping malls considérés comme les plus centraux, les plus emblématiques, dans les villes où elles conduisaient leurs enquêtes. Le choix de comparer a été confirmé 
par des discussions au cours desquelles elles ont identifié des éléments de contexte qu'il était pertinent de mettre en parallèle, notamment le caractère ségrégué des sociétés urbaines étudiées. Le shopping mall constituait un des seuls espaces où les formes de ségrégation genrée et ethno-raciale étaient dans une certaine mesure assouplies. Il y avait donc une compatibilité des matériaux recueillis et des approches à condition de circonscrire l'objet.

Cela n'a pas été le cas pour tous les binômes : certains binômes envisagés ont dû être abandonnés, car ils n'ont pas survécu à l'examen plus minutieux de la comparabilité des matériaux recueillis et/ou des concepts mobilisés. Une comparaison avait ainsi été envisagée autour de la constitution de classes moyennes, notamment par des pratiques consuméristes, en Chine et en Arabie Saoudite. Mais les matériaux d'enquête, recueillis par des méthodes différentes, étaient trop dissemblables, et l'application transversale du concept de classes moyennes nétait pas pertinente. Pour qu'un binôme soit envisageable, il fallait qu'il permette un échange au-delà d'un plus petit dénominateur commun, afin de ne pas enlever toute son épaisseur à lobjet mis en comparaison.

\section{Négocier les concepts, faire émerger des questionnements communs}

Une fois les binômes formés, l'étape suivante a consisté à s'échanger des retranscriptions d'entretiens et des extraits de journaux de terrain, mais aussi des notes de lecture. Chaque binôme discutait ensuite des pistes qui ressortaient de la lecture des documents fournis par l'autre membre, au cours de plusieurs demi-journées entre lesquelles la discussion continuait par courriers électroniques, sur une période d'environ trois mois.

Un apport important de la démarche a été de faire émerger des questionnements communs au prisme desquels porter un regard neuf sur le matériau déjà recueilli. Ainsi, les discussions entre les deux chercheuses du binôme 1 ont permis de dégager trois problématiques communes dont deux relativement classiques au regard des travaux sur les shopping malls (étude des sociabilités / normes consuméristes) ainsi qu'une troisième beaucoup plus originale autour du shopping mall comme espace de promotion d'un imaginaire d'unité et de modernité nationale. En fait, chaque chercheuse avait auparavant ébauché cette piste d'interprétation, puis l'avait abandonnée, la trouvant incongrue. Cela semblait en quelque sorte un aspect trop particulier au contexte étudié pour être réellement creusé. Or les simila- 
rités entre les matériaux recueillis se révélaient saisissantes : dans les deux cas, les images d'unité nationale étaient utilisées dans le cadre de stratégies marketing par les propriétaires des shopping malls, dont les statuts étaient ambigus, entre public et privé, pour des raisons différentes ${ }^{2}$. Les shopping malls pouvaient être des espaces de mise en scène d'une philanthropie ou d'un folklore nationaux, voire de cérémonies officielles (fête nationale). Ils rassemblaient de fait des promeneurs et clients positionnés différemment dans les hiérarchies sociales de genre, de classe, d'ethnicité (pour Port Louis) et de nationalité (pour Riyad).

Prendre conscience de cette similarité a poussé le binôme à un travail conceptuel pour tenter d'exploiter cette piste. Dans le cadre d'une démarche comparative a posteriori, le processus d'écriture, destiné à produire un texte organisé par entrées analytiques (et non par cas), a une place centrale dans la mesure où il devient le lieu de la négociation entre les deux chercheuses. En l'occurrence, elles ont dû tenter de se mettre d'accord sur l'usage quelles feraient de concepts très discutés comme celui d'imaginaire national. Cela les a poussées à échanger des notes et conseils de lecture en pulvérisant les cloisonnements en termes de disciplines et d'" aires culturelles ». Si les chercheurs travaillant sur l'Ile Maurice incluent souvent les publications sur l'Afrique du Sud dans leur bibliographie, ce n'est pas le cas de la plupart des chercheurs travaillant sur la péninsule Arabique, qui sont plutôt incités à lire sur le "monde arabe ». Or, pour les problématiques qui intéressaient ce binôme en termes de ségrégations, les analyses des malls sud-africains étaient particulièrement éclairantes. Par ailleurs, les recherches féministes postcoloniales sur le Moyen-Orient ont produit des réflexions intéressantes concernant les concepts de modernité et de nation. Celles-ci ont alimenté les discussions, parfois vives, au sein du binôme : ainsi, une participante souhaitait parler de cohésion nationale, tandis que l'autre refusait une telle notion et analysait plutôt son matériau en termes d'imaginaire national. Chacune devait alors consolider son interprétation et ses arguments scientifiques en faveur de tel concept, ce qui était heuristique. Le fait que ces débats entre chercheurs aient lieu au moment d'analyser le matériau, c'est-à-dire après la réalisation de l'enquête, mais en amont de l'écriture, nous paraît un autre apport du comparatisme a posteriori, et ce, même si les négociations n'ont pas toujours abouti. Dans le temps imparti pour rédiger la communication, la solution de mettre fin à un désaccord était parfois de couper le passage problématique. Ça a été

2. A Port-Louis, le mall appartient à moitié à un ministère d'Etat, à moitié à une société privée. A Riyad, le mall appartient à un membre de la famille royale. 
le cas par exemple sur certains usages du concept de modernité à propos desquels les deux participantes n'ont pas pu s'accorder.

Les conclusions de ce binôme montrent qu'il est analytiquement pertinent de comparer certains processus, liés, dans ce cas, à la circulation transnationale de modèles urbanistiques, dans des contextes nationaux dont la comparaison pouvait sembler a priori improbable. C'est bien parce que les deux chercheuses avaient travaillé de manière inductive sur un seul contexte que la confrontation a posteriori de leurs données d'enquête et de leurs interprétations leur a permis de rapprocher ces contextes apparemment incomparables.

Les discussions conceptuelles peuvent aussi intervenir au moment de déterminer ce qui est comparable dans le matériau recueilli, comme dans le cas du binôme 2. Si un questionnement de départ commun aux deux chercheuses portait sur la façon pertinente de qualifier les mobilisations que chacune étudiait - mobilisations « identitaires ", " communautaires ", " minoritaires ", « ethniques " ? -, les populations composant ces mobilisations étaient différentes : l'une étudiait les mobilisations de Portoricains aux Etats-Unis et d'Antillais en France et l'autre des mobilisations antiracistes à dimension ethno-raciale en France et en Allemagne (voir Célestine, 2009 ; Laplanche-Servigne, 2011). Pourtant, elles se sont retrouvées, physiquement, sur un même terrain d'enquête : la "Marche des Libertés ", manifestation commémorant l'abolition de l'esclavage, organisée à Paris le 10 mai 2008. A l'issue de plusieurs phases de discussion, c'est la comparaison a posteriori de ce terrain commun - mais mené séparément - qui a semblé la meilleure stratégie pour confronter leurs interprétations et en tirer de nouveaux enseignements pour analyser leurs enquêtes comparatives initiales (France/Etats-Unis pour l'une, France/Allemagne pour l'autre). Dans une première phase dialogique, les deux chercheuses ont d'abord tâché de confronter toutes les mobilisations à dimension ethno-raciale qu'elles étudiaient dans le cadre de leurs comparaisons internationales respectives. Le binôme a alors constaté que malgré les différences, selon le contexte national, des structures d'opportunité politiques saisies par les acteurs mobilisés, un point commun apparaissait : ces mobilisations oscillaient entre revendications culturelles particulières et revendications en vertu d'un statut de minorisés dans leur société. Plus précisément, toutes ces mobilisations apparaissaient informées par la perception de citoyennetés ambivalentes, conduisant leurs initiateurs à se mobiliser, en dépit d'un statut de citoyens, selon des modes d'identification ethno-raciaux (par exemple « en tant que Noirs »). Mais les deux chercheuses utilisaient 
des concepts différents pour qualifier les acteurs des mobilisations : l'une parlait de " migrants-citoyens » pour les Antillais et Portoricains, tandis que l'autre employait le terme de « victimes » (de discriminations raciales) revendiqué dans certains cas par les groupes enquêtés.

Afin de dépasser ces qualifications divergentes, elles ont décidé de focaliser leur comparaison sur la part de terrain qui leur était commune, concernant la commémoration de l'esclavage en France. Elles se sont demandé comment des interrogations initiales en partie distinctes, explorées en enquêtant sur des mobilisations différentes, pouvaient néanmoins conduire à un même lieu d'observation. Elles ont cumulé directement leurs données respectives sur un même terrain d'enquête, ce qui n'est pas si courant, même si la production de résultats en sciences sociales est toujours d'une manière ou d'une autre le résultat d'un processus cumulatif. La comparaison des matériaux recueillis sur ce terrain d'enquête commun a mis en lumière une similitude qu'elles n'avaient jusqu'ici pas problématisée de façon aussi nette : tous les groupes étaient composés (majoritairement) de "nationaux » (et non d'immigrés), qui se mobilisaient, dans le cadre de cette marche, en tant que « victimes » (descendantes d'esclaves). C'est ainsi que la comparaison a fait saillir l'hypothèse suivante : le « télescopage » de l'action d'associations mobilisées au départ principalement sur des questions de mémoire d'une part, et d'autre part d'une organisation axée prioritairement sur la lutte contre les discriminations raciales, résultait d'une appropriation par ces différents acteurs du contexte politique français du milieu des années 2000 marqué par la thématisation de la mémoire et de la reconnaissance des victimes. C'est dès lors la mobilisation d'un statut de « victime " (de discrimination raciale et/ou en tant que descendants de victimes de l'esclavage) qui apparaissait, dans cette marche, comme le point commun pertinent à analyser entre les différentes associations présentes. Cette analyse rendait ainsi saillante aux yeux des deux chercheuses la spécificité du contexte politique français, pour comprendre la logique de construction de mobilisations à dimension ethno-raciale, par rapport aux autres contextes nationaux au cœur de leurs recherches, qu'il s'agisse des Etats-Unis ou de l'Allemagne ${ }^{3}$.

On voit bien, par ces deux cas, comment le comparatisme a posteriori a permis de parvenir, à l'issue de discussions au sein des binômes, à de nou-

3. Une telle convergence n'a pas été observée en Allemagne ou aux Etats-Unis, où l'espace des mobilisations à dimension ethno-raciale apparaissait plus segmenté, en l'absence d'un cadre d'opportunités politiques commun tel que celui des politiques mémorielles et de reconnaissance des victimes en France au milieu des années 2000 . 
veaux questionnements, voire à des hypothèses communes, en confrontant des matériaux d'enquêtes produits sur la base d'objets construits différemment. C'est un atout de cette démarche que de travailler avec des approches déjà problématisées en fonction de la spécificité des contextes étudiés. Cela permet de ne pas restreindre l'ouverture d'une réflexion inductive fondée sur le matériau empirique recueilli, comme c'est souvent le cas pour les comparaisons construites en amont. En même temps, cette démarche dispose d'un avantage par rapport à une monographie : elle s'ouvre à un regard extérieur un peu décalé.

\section{Ine approche enrichie de la monographie}

Quand les binômes constitués n'ont pas permis d'aboutir à des hypothèses communes, la pratique de la discussion, puis de l'écriture en binôme a permis d'enrichir considérablement les monographies des participants, tant du point de vue de la réflexivité sur la démarche d'enquête que des concepts mobilisés.

Les pratiques réflexives sont actuellement encouragées en sciences sociales, qu'elles soient envisagées comme une manière de construire l'objectivité ou dans la perspective féministe du savoir situé (pour le cas français, voir notamment Naudier et Simonet, 2011). Or l' " auto-analyse » est un processus ardu qui laisse toujours des points aveugles. A défaut de débattre entre chercheurs au moment même de l'enquête pour mettre à jour les ébauches d'interprétation intuitives, souvent non explicitement formulées, qui guident les stratégies adoptées sur le terrain (Olivier de Sardan, 1995), le comparatisme a posteriori constitue une première avancée pour une approche plus collective permettant de gagner en réflexivité sur les stratégies interprétatives après l'enquête, mais en amont de l'écriture. Le processus de discussion en binôme, nécessaire pour produire une comparaison a posteriori, permet ainsi d'enrichir la pratique de la réflexivité non seulement sur le rapport à l'objet de recherche, mais aussi sur les pratiques d'enquête et d'interprétation. Il permet dès lors de bénéficier d'un des apports possibles de la recherche collective (Wrede et al., 2006), mais sans que le processus soit aussi contraignant, en termes de coûts matériels et de disponibilité, et avec le gain d'un regard extérieur.

Dans le cas d'une enquête ethnographique, la question de la réflexivité se pose de manière particulière, puisque le matériau d'enquête est produit dans le rapport intersubjectif entretenu par les chercheurs avec leurs en- 
quêtés ${ }^{4}$. Etant donné ce mode de production des données, il est rien moins quévident de réfléchir seul à la question des émotions ressenties et partagées avec les enquêtés, à celle de l'empathie, notamment quand il s'agit d'enquêtés qui luttent pour des droits ou une reconnaissance qui semblent - subjectivement - légitimes. Les chercheurs ont dans les faits rarement l'occasion de mettre leurs retranscriptions d'entretien et des extraits de leur journal de terrain à la disposition d'autres chercheurs, incités par l'écriture d'un texte commun à les lire sérieusement. Le travail en binôme a ici permis de produire une réflexivité sur le mode de production des données et sur la construction de leurs interprétations. Pour des raisons de concision, nous nous appuierons ici uniquement sur le cas du binôme 3 .

L'objectif des deux chercheuses était d'identifier des régimes de contraintes à l'action collective qui seraient spécifiques aux modes autoritaires de gouvernement à partir d'une étude de l'émergence d'espaces d'expression plus libres à La Havane et à Pékin depuis la fin des années 1980. Au cours de leurs échanges préparatoires, les deux chercheuses se rendirent compte que la trop forte élasticité (Sartori 1991) de la notion d' " espaces d'expression » empêchait d'atteindre cet objectif. Leurs objets - un échantillon d'internautes pékinois des nouvelles classes moyennes urbaines pour l'une et des collectifs de jeunes artistes et intellectuels contestataires havanais, actifs dans les marges de la sphère culturelle, pour l'autre - étaient trop dissemblables. En revanche, les discussions de ce binôme se révélèrent fructueuses à un autre niveau, celui de l'interprétation des données.

La chercheuse qui s'intéressait aux internautes chinois avait surtout conduit des entretiens et des observations en ligne sur des chats et des forums. Celle qui étudiait les collectifs havanais avait choisi une méthode ethnographique d'immersion longue qui impliquait de partager le plus possible la vie des enquêtés. Les membres du binôme choisirent donc de se pencher sur les discours de leurs enquêtés afin de travailler des matériaux comparables. Elles échangèrent des transcriptions d'entretiens et les notes prises lors d'échanges plus informels avec leurs enquêtés. Cette mise en commun des données entraîna de vifs échanges entre les deux chercheuses du fait de leurs perspectives opposées sur l'importance des émotions dans la prise de parole en contexte autoritaire. La chercheuse étudiant les contestataires cubains interprétait la multiplicité des discours des enquêtés selon la situation d'énonciation comme un effet de la peur qu'ils ressentaient. Cette interprétation était

4. Si l'intersubjectivité est en général inexistante dans la méthode quantitative, nous sommes convaincues qu'une plus grande réflexivité est nécessaire dans l'ensemble des travaux de sciences sociales, quelles que soient les méthodes utilisées. 
liée à sa propre expérience d'une peur omniprésente d'être arrêtée, interrogée et expulsée. Si la chercheuse étudiant les internautes pékinois ne remettait pas en question l'existence d'émotions négatives - présentes en Chine - elle questionnait la place centrale que leur attribuait la première chercheuse au détriment d'autres pistes interprétatives qui lui paraissaient tout aussi pertinentes. Il lui semblait, à la lecture des entretiens et des notes partagées, que les contestataires cubains, comme les internautes chinois, disposaient de savoirs intuitifs en matière de limites du possible et du jouable en termes de critiques à l'encontre du gouvernement, sans que des règles explicites soient le plus souvent énoncées par les autorités. D'après elle, certaines lignes rouges étaient assez largement perçues comme telles et donc rarement franchies. A l'inverse, pour l'autre chercheuse, l'incertitude était permanente.

Cette confrontation amena cette chercheuse à aller au bout de sa logique argumentative et donc à défendre ce quelle identifiait comme le point de vue des enquêtés cubains, et qui était en réalité le sien. Elle prit conscience que ses arguments nétaient pas fondés sur une analyse distanciée des propos multiples et contradictoires des enquêtés, mais sur la forte empathie non réfléchie qu'elle avait développée envers eux, car elle avait vécu son immersion dans le contexte autoritaire cubain de manière particulièrement anxiogène. Elle avait par conséquent sur-interprété les éléments de discours qui soulignaient le rôle de la peur dans la délimitation des formes d'action collective des enquêtés. C'est le regard extérieur de son binôme qui lui permit de situer ses modes de perceptions, en les reliant au contexte pluraliste de sa socialisation politique. Cette socialisation spécifique l'avait conduite à accompagner avec anxiété les enquêtés dans leurs performances de rue et à envisager la plupart de leurs actions comme des formes de confrontation avec les autorités cubaines. Ce regard extérieur l'incita à distinguer entre des modes d'action partiellement routinisés, autrement dit relativement prévisibles pour les autorités et donc généralement tolérés, et des « coups » dont les effets étaient alors très incertains. Cette réflexion partagée l'amena finalement à mieux prendre en compte les éléments qui suggéraient l'existence de modes de négociation avec les autorités, éléments quelle avait en partie écartés, car ils allaient contre son appréhension dichotomique des relations entre autorités et groupes contestataires. Cet exemple nous montre que la place des chercheurs dans le processus de constitution intersubjective du matériau ethnographique et de son interprétation est l'un des enjeux fondamentaux de la pratique de la comparaison a posteriori, qu'il faut explorer plus avant, car cette pratique amène à dénaturaliser la place occupée par les chercheurs sur leur terrain et à questionner l'utilisation du matériau recueilli ainsi que la mise en forme des interprétations. Cette dénaturalisation est plus forte encore que 
dans une recherche à deux sur le même terrain, car la non implication de l'autre chercheur du binôme dans la production du matériau lui permet de déconstruire les interprétations immédiates formées à chaud et qui, bien souvent, modèlent déjà les pistes d'analyse explorées par la suite.

Au-delà de la question de la réflexivité, la production de textes en binôme a permis d'enrichir les monographies initiales des participants. Si les papiers produits nont pas été publiés en tant que tels, en revanche, les hypothèses développées ont été reprises dans les travaux individuels. Même pour les binômes qui ont peiné à dégager des hypothèses communes, la tentative a été en soi enrichissante pour irriguer les recherches doctorales en cours. Lexpérience du binôme 3 est particulièrement révélatrice de cet autre apport du comparatisme a posteriori. Dans ce cas, c'est justement le rapprochement difficile entre deux objets à comparabilité molle (les espaces d'expression en contexte autoritaire), qui a permis aux deux chercheuses impliquées de s'interroger autrement sur les relations entre enquêtés et autorités, autrement dit sur le rapport des enquêtés au politique. La question de la « politisation » avait été tranchée de façon antagonique, notamment du fait des perspectives adoptées sur leur objet. A Pékin, la chercheuse qui s'intéressait à la prise de parole en ligne analysait la politisation des internautes ordinaires observés comme individuelle, minoritaire et souvent involontaire quand elle avait lieu (voir Arsène, 2011). A La Havane, la chercheuse qui enquêtait sur des collectifs contestataires interprétait leur action comme de nature politique sans être revendiquée comme telle (voir Geoffray, 2012). Or, ces choix antagoniques (politisation évidente / absence de politisation) leur parurent étonnants au regard de leurs objets, relativement proches, et de leur objectif partagé de désenclaver les travaux sur la prise de parole (cas pékinois) ou l'action collective (cas havanais) en contexte autoritaire, en maniant des travaux construits presque exclusivement, sur ces questions-là, en contexte démocratique.

Les lectures croisées de transcriptions d'entretiens et de notes avaient fait émerger une sorte de malaise interprétatif chez les deux chercheuses, car chacune pointait chez l'autre les contradictions liées à la volonté de rendre lisible une parole multiple et souvent fortement contradictoire. Ce dialogue leur permit dans un premier temps de prendre de la distance vis-à-vis des mots des enquêtés et dans un second temps de déporter l'attention vers les interactions entre les actions menées, les discours tenus par les enquêtés sur ces actions et les réactions des autorités vis-à-vis de ces actions. Il en ressortit que les prises de parole (des internautes chinois) ou les actions de rue (des contestataires cubains) pouvaient avoir des effets $a$ posteriori sur des publics ou sur les autorités, qui nétaient ni toujours in- 
tentionnels ni toujours anticipés. Dans le cas chinois, le processus cumulatif des prises de parole individuelles sur des forums Internet créait en effet des formes inédites et éphémères de coordination, qui pouvaient aboutir à la construction publique de scandales. La répression parfois forte, par les autorités chinoises, de ces prises de parole individuelles et spontanées confirmait cette analyse en montrant qu'elles nétaient pas anodines. A l'inverse, les discours des enquêtés cubains sur les mises en scène publiques d'actions contestataires dans le but de perturber l'ordre social révolutionnaire étaient souvent contredits par le laissez faire que pratiquaient à leur égard les autorités cubaines. Si ces dernières n'interprétaient pas les actions des collectifs comme politiques, alors l'étaient-elles?

Ces échanges menèrent les deux chercheuses à infléchir leurs positions respectives et à mieux prendre en compte les ambivalences et les contradictions propres aux discours ainsi qu'aux pratiques des enquêtés. Si ce dialogue comparatif ne mena pas à des analyses comparatistes plus poussées, il fut cependant essentiel dans l'orientation ultérieure de chaque thèse, en conduisant à interroger les effets des actions menées et leur réception par des publics et par les autorités pour comprendre le phénomène de la politisation, plutôt que sur les seules intentions des acteurs étudiés. Dans le cas de la chercheuse sur les contestataires cubains, ce moment comparatif fut essentiel, car il l'amena à s'intéresser aux contradictions observées en tant que telles et partant, à faire de l'ambiguïté - celles des collectifs comme celle des autorités, dans les paroles comme dans les pratiques - le fil conducteur de sa thèse.

\section{Conclusion : un comparatisme transitoire}

Le comparatisme a posteriori a finalement eu plusieurs types d'apports, cumulés ou non selon les binômes. Un premier apport est l'aspect dialogique, le fait de questionner en binôme les analyses produites par l'interprétation du matériau de terrain. Ce dialogue est heuristique à plusieurs égards : premièrement, il permet de déconstruire l'exceptionnalité supposée de certains cas en les confrontant avec d'autres. Deuxièmement, il peut attirer l'attention des chercheurs sur des éléments qu'ils auraient négligés : c'est le « rapprochement heuristique » au sens de Paul Veyne (1998 : 170171). Troisièmement, il permet une distanciation salutaire par rapport à l'objet et une réflexivité sur les stratégies d'enquête et d'interprétation.

Le second apport a été, pour certains binômes, de parvenir à produire des avancées analytiques, sans que leur démarche comparatiste ne corresponde 
pour autant à celle que Kocka (2003) qualifie d'« analytique » (tester des rapports de causalité). La comparaison de deux cas a permis de mettre en exergue des récurrences révélatrices de la circulation de normes et de modèles ainsi que des phénomènes de convergence - dimension au centre du renouvellement des approches comparatives a priori (Hassenteufel, 2000 ; 2005). Ainsi le binôme 1 a mis en évidence la circulation de normes et de modèles qui produit des effets comparables dans des contextes très différents, tandis que le binôme 2 a permis l'élaboration d'une catégorie de représentation (les victimes) partagée par des groupes sociaux distincts dans un même contexte politique et social. Cette confrontation de deux enquêtes ne vise alors pas à l'établissement de taxinomies, mais à un enrichissement mutuel des concepts et des questionnements et à la mise en évidence de processus en partie liés les uns aux autres sans pour autant écraser les spécificités de chaque contexte. La démarche permet ainsi de gagner en originalité des questionnements et en finesse d'analyse. Elle pourrait aboutir à des résultats publiables qui s'apparenteraient à ceux d'une enquête collective multi-sites tout en amoindrissant le risque - par rapport au comparatisme a priori - que les grilles d'observation et d'analyse soient plaquées sur chaque contexte et en négligent les spécificités.

Si les apports du comparatisme a posteriori pourraient éventuellement devenir le point de départ d'autres enquêtes, menées selon une démarche comparative plus conventionnelle (ce que nous qualifions de comparaisons " a priori »), il constitue dans tous les cas un moyen de mettre au jour de nouvelles interprétations et hypothèses pour les chercheurs impliqués. C'est dans ce sens que cette démarche peut être dite transitoire, dans la mesure où elle mène les participants à franchir une étape, qu'il s'agisse d'infléchir des recherches en cours en leur donnant une nouvelle orientation comparative ou de questionner les interprétations les plus intuitives par leur mise à lépreuve dans le dialogue comparatif. Elle contribue enfin, par sa méthodologie reposant sur le croisement systématique des regards, à questionner et transgresser différentes frontières encore largement présentes dans les sciences sociales : entre « monographie » et " approche comparative », entre " savoir disciplinaire général » et « savoir spécifique aux aires culturelles », et enfin entre différentes échelles d'observation et d'analyse ${ }^{5}$.

5. Nous remercions très chaleureusement les co-organisatrices de la journée d'étude, Séverine Arsène et Yasmine Berriane, les participants des différents binômes, Amin Allal, Irene Bono, Audrey Célestine, Guillaume Gourgues, Yasushi Iwabuchi, Alice Mazeaud, Foued Nasri, Julie Peghini et Christophe Premat, ainsi que les discutants, Richard Banégas, Jean-François Bayart, Loïc Blondiaux, Manlio Cinalli, Olivier Dabène, Camille Hamidi, Béatrice Hibou et Monika Salzbrunn. Les opinions exprimées dans l'article n'engagent bien sûr que les auteures. 


\section{1. références}

Arsène S., 2011. Internet et politique en Chine, Paris, Karthala.

Bayart J.-F., 1992. Le politique par le bas en Afrique noire, Paris, Karthala. Briquet J.-L., Sawicki F., 1989. L'analyse localisée du politique, Politix, vol. 2, n $7,6-16$.

Detienne M., 2000. Comparer l'incomparable, Paris, Seuil.

Célestine A., 2009, Mobilisations collectives et constructions identitaires. Le cas des Antillais en France et des Portoricains aux Etat-Unis, Thèse de doctorat, IEP de Paris.

Geoffray M.-L., 2012, Contester à Cuba, Paris, Dalloz.

Giraud O., 2003. Le comparatisme contemporain en science politique : entrée en dialogue des écoles et renouvellement des questions, in Lallement M., Spurk J. (dir.), Stratégies de la comparaison internationale, Paris, CNRS Editions, 87-106.

Hassenteufel P., 2000. Deux ou trois choses que je sais d'elle : remarques à propos d'expériences de comparaisons européennes, in C.U.R.A.P.P., Les méthodes au concret, Paris, P.U.F-C.U.R.A.P.P., 105-124.

Hassenteufel P., 2005. De la comparaison internationale à la comparaison transnationale : les déplacements de la construction d'objets comparatifs en matière de politiques publiques, Revue Française de Science Politique, vol. 55, n $\mathrm{n}^{\circ} 155$, février, 113-132.

Kocka J., 2003. Comparison and beyond, History and theory, vol. 42, $\mathrm{n}^{\circ} 1$, février, 39-44.

Laplanche-Servigne S., 2011. De l'expérience du racisme à sa politisation. Mobilisations et représentation des «victimes» en France et en Allemagne aujourd'hui, thèse de doctorat, IEP de Paris.

Le Renard A., 2011. Femmes et espaces publics en Arabie Saoudite. Paris, Dalloz.

Naudier D., Simonet M. (dir.), 2011. Des sociologues sans qualité ? Pratiques de recherche et engagements, Paris, La Découverte.

Olivier de Sardan J.-P., 1995. "La politique du terrain ", Enquête, mis en ligne le 1 février 2007. URL : http://enquete.revues.org/document263. html.

Peghini J., 2009. Les impasses du multiculturalisme. Politique, industries et tourisme culturel à l'île Maurice, thèse de doctorat, Paris 8 (Thèse remaniée à paraître aux Presses Universitaires de Vincennes en 2013).

Picard E. (dir.), 2006. La politique dans le monde arabe, Paris, Armand Colin.

Revel J., 1996. Jeux d'échelles : la micro-analyse à l'expérience, Paris, Seuil. 
Sartori G., 1991. "Comparing and miscomparing ", Journal of Theoretical Politics, vol. 3, n 3, 243-257.

Thiriot C., Marty M. et Nadal E., 2004. Penser la politique comparée. Un état des savoirs théoriques et méthodologiques, Paris, Karthala.

Veyne P., 1976. L'inventaire des différences, Paris, Seuil.

Veyne P., 1998 (1971). Comment on écrit l'histoire, Paris, Points.

Vigour C., 2005. La comparaison dans les sciences sociales. Pratiques et méthodes, Paris, La Découverte.

Wrede S. et al., 2006. Decentred comparative research: Context sensitive analysis of maternal health care, Social Science E Medicine, n63, 29862997.

Marie-Laure Geoffray (marie-laure.geoffray@univ-paris3.fr) Maîtresse de conférences en science politique IHEAL-Paris 3

Amélie Le Renard (amelie.lerenard@gmail.com) Chargée de recherche CNRS en sociologie CMH-PRO (CNRS-EHESS-ENS)

Soline Laplanche-Servigne (solinels@hotmail.com) Université de Tours, CEE - Sciences Po 